\title{
Optical, Photoelectrochemical and Impedance Studies on Poly 3,3' Dibromo, 2,2' Bithiophene: Effect of Bromine as a Substituent at C-3 Position in 2,2' Bithiophene Molecules
}

\author{
Kasem K. Kasem ${ }^{1}$, Houria Sadou ${ }^{1}$, Anthony W. Jeffers ${ }^{1}$ \\ ${ }^{1}$ Indiana University Kokomo, School of Sciences, Kokomo, IN, USA \\ Correspondence: Kasem K. Kasem, Indiana University Kokomo, School of Sciences, Kokomo, IN, USA. \\ E-mail: kkasem@iuk.edu
}

Received: October 23, 2018 Accepted: November 15, 2018 Online Published: November 19, 2018

doi:10.5539/ijc.v10n4p83

URL: https://doi.org/10.5539/ijc.v10n4p83

\begin{abstract}
Thin films of poly 3, 3' dibromo, 2,2' biththiophene (PDBrBTh) and poly 2,2' biththiophene (PBTh) on fluorine doped tin oxide electrode (FTO) were subjected to optical, electrochemical and impedance studies in various aqueous electrolytes. Both polymers showed electrochromic properties, where the neutral film at $-1.0 \mathrm{~V}$ vs $\mathrm{Ag} / \mathrm{AgCl}$ shows red color, and the oxidized (doped) film at positive potentials shows blue color. Optical studies indicated that PDBrBTh showed a greater optical band gap than that of PBTh. Our studies also showed that the presence of bromine as a substituent at the C-3 position of the thiophene ring causes a greater ionization potential (IP) than PBTh. PDBrBTh also shows closer values of refractive index $(\mathrm{n})$, and real $\left(\varepsilon_{\mathrm{r}}\right)$ and imaginary $\left(\varepsilon_{\mathrm{i}}\right)$ dielectric constants to that of PBTh at photon energies between $2.0 \mathrm{eV}$ and $3.0 \mathrm{eV}$. At lower photon energy PDBrBTh showed lower optical conductivity than Br-free PBTh. Study also indicated that PDBrBTh is much less photoactive that PBTh. Electrochemical impedance spectroscopic studies (EIS) revealed that PDBrBTh possesses less relaxation time, and less double layer capacitance $\left(\mathrm{C}_{\mathrm{dl}}\right)$ in dark and under illumination.
\end{abstract}

Keywords: photoactive polymers, impedance, photoelectrochemistry, optical studies

\section{Introduction}

Regardless of their state, in solution, amorphous, crystalline, particle, thin solid or gel forms, photoactive organic polymers undergo changes in their physical properties upon illumination. These changes could be due to alterations in their molecular geometry and possible electronic configurations. These changes can be permanent or reversible. Observable changes include optical, electrochromic and electric properties. Often, these changes can be used in an applied capacity such as building up sensors or transistors.

Examples of these photoactive, intrinsic conducting polymers are polythiophenes and their derivatives (Arif K.et al 2018). Several research works were focused on their noticeable properties such as electrical conductivity, redox behavior, electrochromic response and their extensive photoelectrical behavior (Kohsuke K. et al, 2012, Buket B.C, 2017, Chung-Wen et al 2018, Yen Wei et al 1991, and Druy M.A et al 1983). These polymers have a great potential for industrial application in many areas. In recent years, many novel devices based on conducting polymers like organic thin film transistors (Arif K.et al 2018), solar cells and batteries (Kohsuke K. et al, 2012), and electrochromic devices have been built (Buket B.C, 2017, Chung-Wen et al 2018, Yen Wei et al 1991, and Druy M.A et al 1983).

Electropolymerization of the organic monomer on the electrode surface can generate diverse uniform/stable structures. Several applications were based on the outcome of immobilization using electropolymerization, such as advanced ion beam techniques (Teare D.O.H et al 2002), self-assembly processes [Deckers G. 1997, Zhang X. et al 1999), chemical attachment of long-chain molecules to a surface [Aulman A. 1996), and the cross-linking of a random copolymer film (Ryu D.Y. et al 2005).

Creation of photoactive polythiophene assembles was achieved by several methods, most importantly the electrochemical method. The advantage of this method is that different monomers can be combined in defined ratios to create a polymer with desired film characteristics and band gap.

Changing the 2-2 bithiophene structure by adding a functional group that may have electron withdraw/donating (W/D) effects on the main thiophene ring that can alter its intrinsic properties. These electron W/D effects may also alter the 
optical dielectric, photoelectrochemical behavior of the polymer of the generated thiophene derivative (Figure 1).

In this study, we focused on determining how different the physical and chemical properties of poly 3,3' dibromo-bithiophene (PDBrBTh) are from those of poly 2,2 biothiophene (PBTh). In particular, we investigated differences in the absorption spectra, refractive index, dielectric constant, oxidation/reduction potential, and geometric structure. These parameters can shed light on whether light-induced changes are reversible or not.
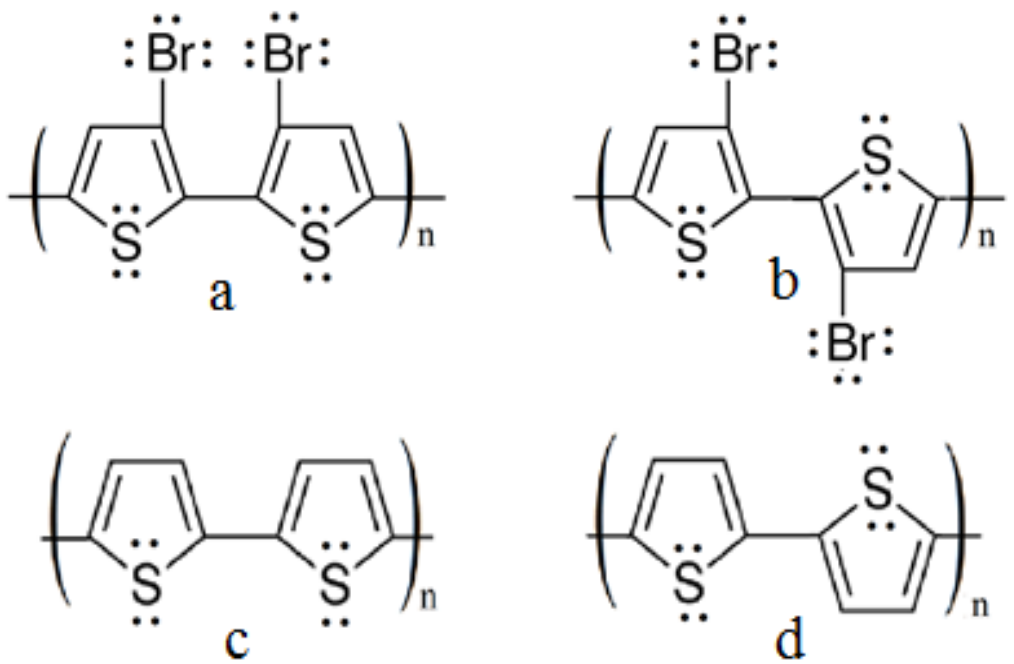

Figure 1. Possible configurations of PDBrBTh (a and b), and PBTh

\section{Experimental}

\subsection{Reagents}

The monomers 2,2` bithiophene (BTh), and 3,3` dibromo thiophene ( $\mathrm{DBrBTh}$ ) were used to prepare their corresponding polymers: poly-2,2 bithiophene (PBTh) and poly-3,3`dibromo thiophene (PDBrBTh), respectively. All of the chemicals used were of analytical grade. Unless otherwise stated, aqueous solutions were prepared using deionized (DI) water. Fluorine-doped tin oxide (FTO) glass was used as substrate for the working electrode.

\subsection{Preparations}

The polymers were prepared using cyclic voltammetry $(\mathrm{CV})$ by repetitive cycling of the substrate electrode (FTO) potential between -0.1 and $0.2 \mathrm{~V}$ vs $\mathrm{Ag} / \mathrm{AgCl}$ in acetonitrile solution containing $10 \mathrm{mM}$ of the monomer and $0.5 \mathrm{M}$ $\mathrm{LiClO}_{4}$.

\subsection{Instrumentation}

A conventional three-electrode cell consisting of a Pt wire as a counter electrode, a $\mathrm{Ag} / \mathrm{AgCl}$ reference electrode, and FTO with surface area $2.0 \mathrm{~cm}^{2}$ as a working electrode was used for electrochemical studies. Photoelectrochemical studies on the thin solid films were performed on the experimental setup as described in previous work (Kasem K.K et al 2018). Optical parameters were calculated based on the steady state reflectance spectra, measured by a Shimadzu UV-2101PC spectrophotometer. An Olympus BX-FL reflected light fluorescence microscope, working with polarized light at wavelengths ranging between 330 and $550 \mathrm{~nm}$ was used to visualize the surface imaging of the film. Irradiation was performed with a solar simulator 300-watt xenon lamp with an IR filter. All measurements were performed at 298 ${ }^{\circ} \mathrm{K}$. A Solartron 2101A was used for EIS studies. A BAS 100W electrochemical analyzer (Bioanalytical Co.) was used to perform the electrochemical studies.

\section{Results and Discussion}

\subsection{Electropolymerization of 3,3`Dibromo Thiophene (DBrBTh)}

Polymer thin films were prepared using an oxidative electropolymerization method by repetitive cycling the potential of FTO (as substrate) at a scan rate $0.1 \mathrm{~V} / \mathrm{s}$ between $-1.00 \mathrm{~V}$ and $1.8 \mathrm{~V} \mathrm{vs} \mathrm{Ag} / \mathrm{AgCl}$ in an electrolyte described in preparation section. The results are displayed in Figure 2. The expanded view in this figure shows a growth of cathodic peak in a potential range $1.2 \mathrm{~V}$ to $1.0 \mathrm{~V}$ vs $\mathrm{Ag} / \mathrm{AgCl}$ upon repeating the scans. These cathodic peaks would appear only if the anodic scan reaches $1.8 \mathrm{~V}$. No polymerization was observed at a voltage lower than this potential. 


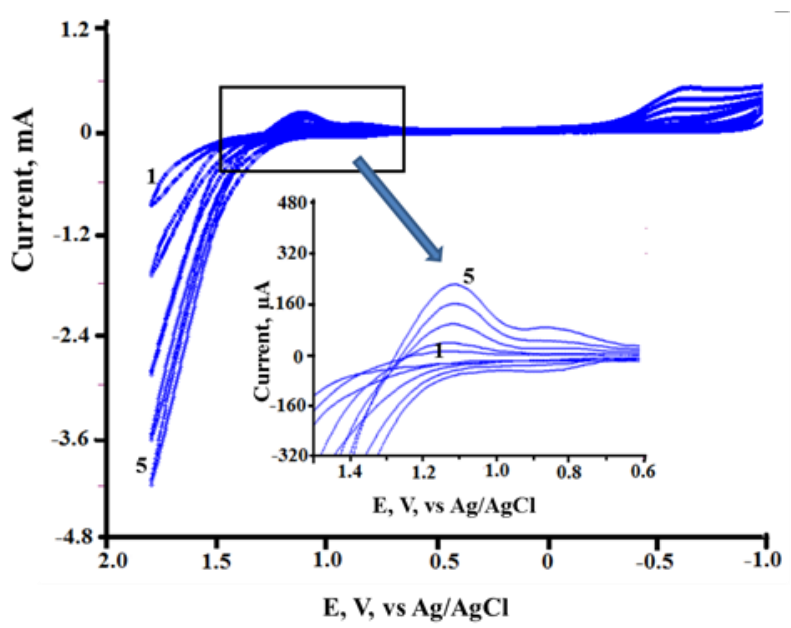

Figure 2. Electropolymerization of $\mathrm{DBrBTh}$ at scan rate $0.1 \mathrm{~V} / \mathrm{s}$ (scan numbers are indicated on the Figure)

\subsection{Electrochromic Behavior}

Both PDBrBTh and PBTh showed electrochromic behavior. Their films were blue at $1.6 \mathrm{~V}$ vs $\mathrm{Ag} / \mathrm{AgCl}$, while at -1.00 $\mathrm{V}$ vs $\mathrm{Ag} / \mathrm{AgCl}$ they were red. The blue color is the complementary color of the broad absorption peak $(\lambda 600-800 \mathrm{~nm})$ (Figure $3 \mathrm{~b}$, and $\mathrm{b}^{\prime}$ ). The red color corresponds to the absorption peak between $\lambda 450-560 \mathrm{~nm}$. We suggest that at $-1.0 \mathrm{~V}$ $\mathrm{vsAg} / \mathrm{AgCl}$ the polymer film is neutral and the polymer film segments follow the structure's pattern shown in Figure $1 \mathrm{~b}$ and $d$. These structures offers the greater level of $\pi$-bond conjugation. Greater conjugated systems have larger absorption peaks and tend to show a redshift (shifted toward the longer wavelength region). Figure 3A-a shows that neutral PBTh offers a more conjugated system than that of the neutral PDBrBTh (Figure B-a'), which also shows an absorption peak in the red range of the spectrum $(\lambda 600-800 \mathrm{~nm})$. This peak is lacking in the absorption spectra of PBTh, indicating that the peak is due to the presence of bromine atoms in the structure. Contrary to that, when the films subjected to the constant potential of $1.4 \mathrm{~V}$ vs $\mathrm{Ag} / \mathrm{AgCl}$, it will be doped with $\mathrm{ClO}_{4}{ }^{-}$, the absorption peaks of PBTh (Figure 3A-b) and of PDBrBTh (Figure 3B-b') underwent blue shift. This indicated that doping the films with electrolyte anions decreased the level of conjugation. We suggest that incorporation of $\mathrm{ClO}_{4}{ }^{-}$causes low level $\pi$-bond conjugation.
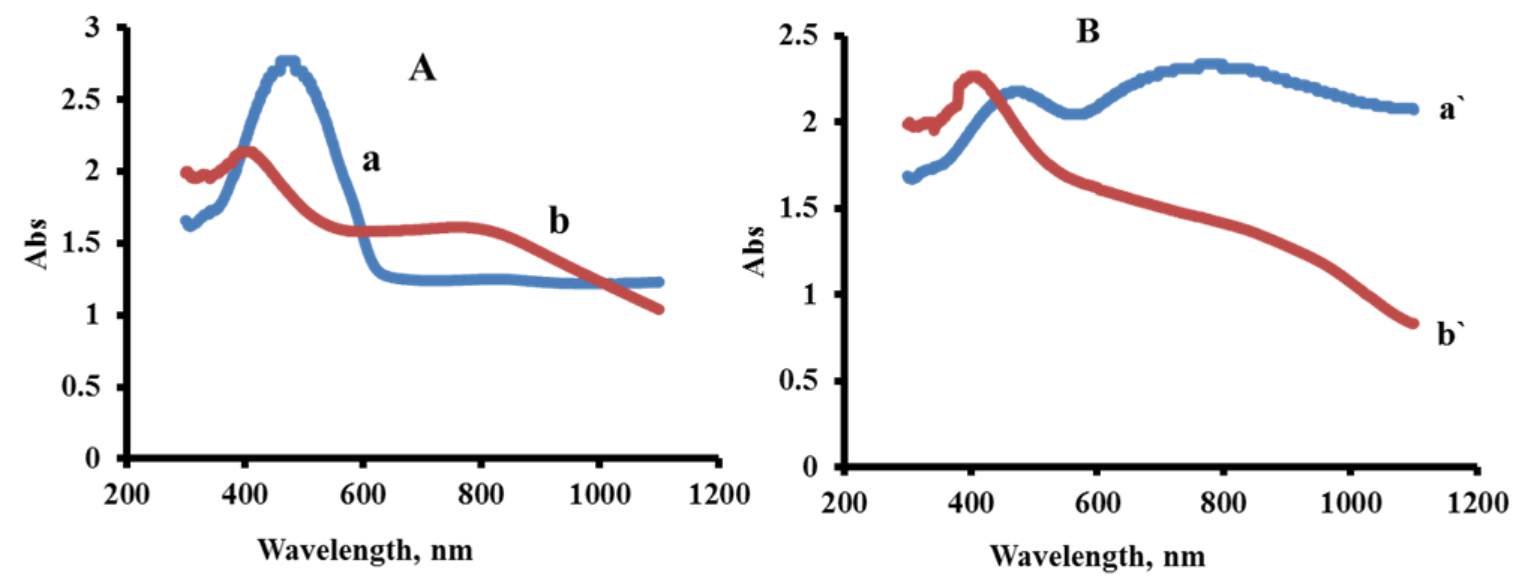

Figure 3. Absorption spectra of A) PBTH $\quad$ B) PDBrBTh, a, a' neutral films at $-1.0 \mathrm{~V} \mathrm{vs} \mathrm{Ag} / \mathrm{AgCl} ; \quad$ b, b' doped films at $1.6 \mathrm{~V}$ vs $\mathrm{Ag} / \mathrm{AgCl}$.

\subsection{Optical Studies}

Parameters such as $\sigma_{\text {opt. }}, \alpha, \mathrm{n}, \varepsilon_{\mathrm{r}}$ and $\varepsilon_{\mathrm{i}}$ have been calculated and plotted as a function of photon energy. The results are displayed in Figures 2, 3, 4 and 5.

\subsubsection{Optical Band Gap Studies}

The UV-VIS absorption spectra of PBTh (Figure 3A-a) and PDBrBTh (Figure 3B-b) show that PDBrBTh possesses 
overlapping peaks, one at $\approx 2.2 \mathrm{eV}$ and one at $\approx 3.0 \mathrm{eV}$, while PBTh possesses a broader peak maximum at $\approx 2.2 \mathrm{eV}$. Figures $3 \mathrm{~B}$ and $3 \mathrm{C}$ were prepared after treatment of the absorption data as plots of $\alpha^{1 / 2}$ vs photon energy (hv) and $\left(\alpha^{*} \mathrm{hv}\right)^{2}$ vs hv, respectively, as described in the previous study [(Tauc J, 1968). The value of $\alpha$ was calculated using a film thickness of $1.0 \mu \mathrm{m}$. Figures 3B and 3C indicated that both PDBrBTh and PBTh show both indirect (Figure 3B) and direct (Figure 3C) band gaps. We suggest that different configurations do exist in the polymer matrices which may create hybrid sub-bands with smaller band gaps between the highest occupied molecular orbitals (HOMO) and lowest unoccupied molecular orbitals (LUMO) of the polymer.
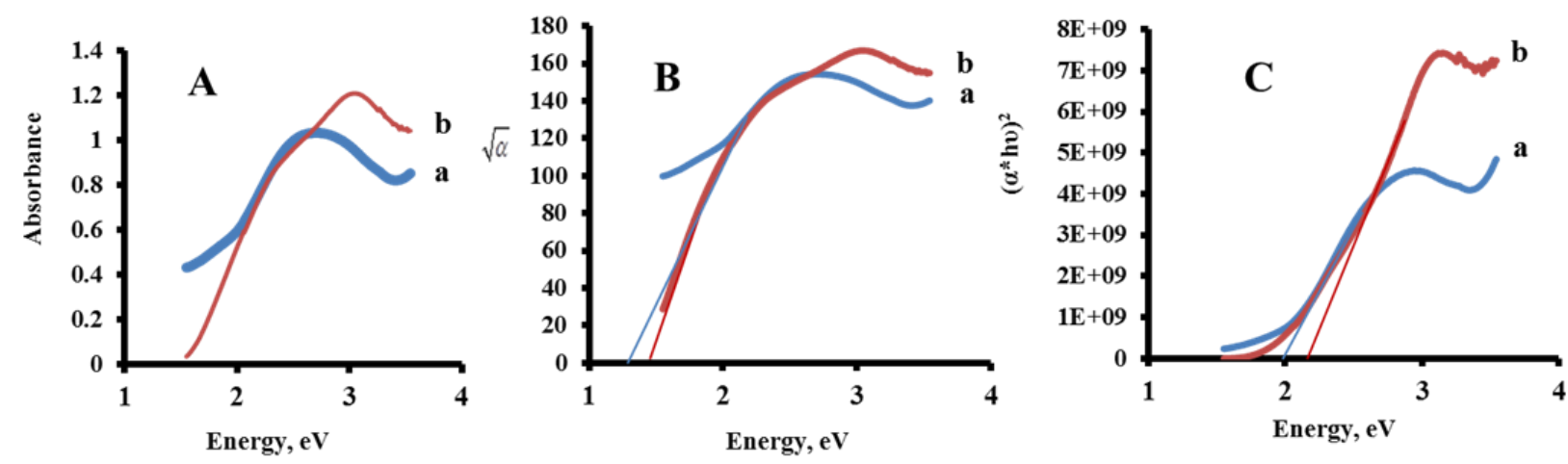

Figure 3. A) Absorption spectra, B) $\alpha^{1 / 2}\left(\mathrm{~cm}^{-1 / 2}\right)$ vs photon energy, and C) $\left(\alpha^{*} \mathrm{hv}\right)^{2}(\mathrm{eV} \cdot \mathrm{cm})^{2}$ vs photon energy for a) PBTh, and b) PDBrBTh

Figure 4, shows that the slopes in the displayed equation correspond to Urbach energy (Urbach F. 1953) which corresponds to $0.824 \mathrm{eV}$ for PBTh and $0.730 \mathrm{eV}$ for PDBrBTh (table 1).

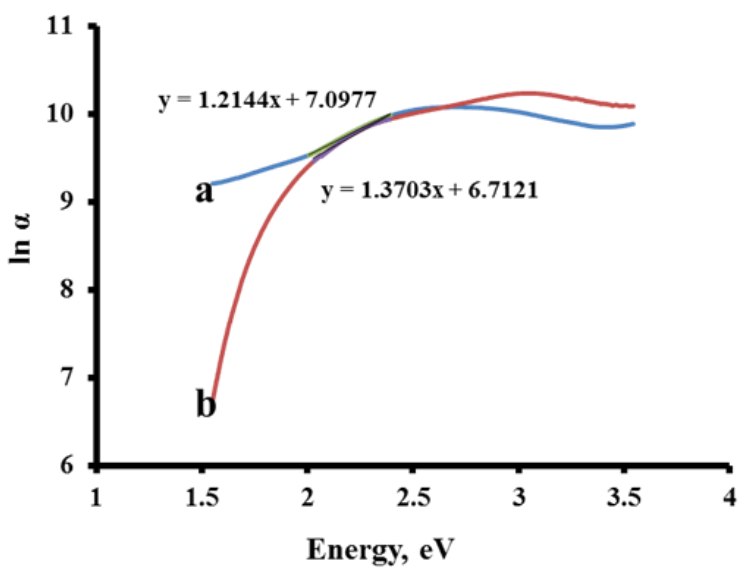

Figure 4. Plot of ln $\boldsymbol{\alpha}$ vs photon energy for a) PBTh and b) PDBrBTh

\subsubsection{Optical Dispersion Characteristics}

To investigate whether these polymers obey a single or multiple oscillation mode, a plot of refractive index (n) or its derivatives vs photon energy Figure 5 was constructed.

a) Refractive index, $n$

Figure 5A displays the plot of refractive index (n) vs wavelength. While PBTh did not exhibit large increase in $n$ over all the studied wavelengths, the value of $\mathrm{n}$ for PDBrBTh noticeably decreased at wavelengths longer than $570 \mathrm{~nm}$. This indicates that PDBrBTh follows the multi-oscillator model (Wemple S.H. et al 1971) at $\lambda$ 's longer $570 \mathrm{~nm}$ while PBTh partially follows the multi-oscillator model $\mathrm{n}$ above $570 \mathrm{~nm}$. 

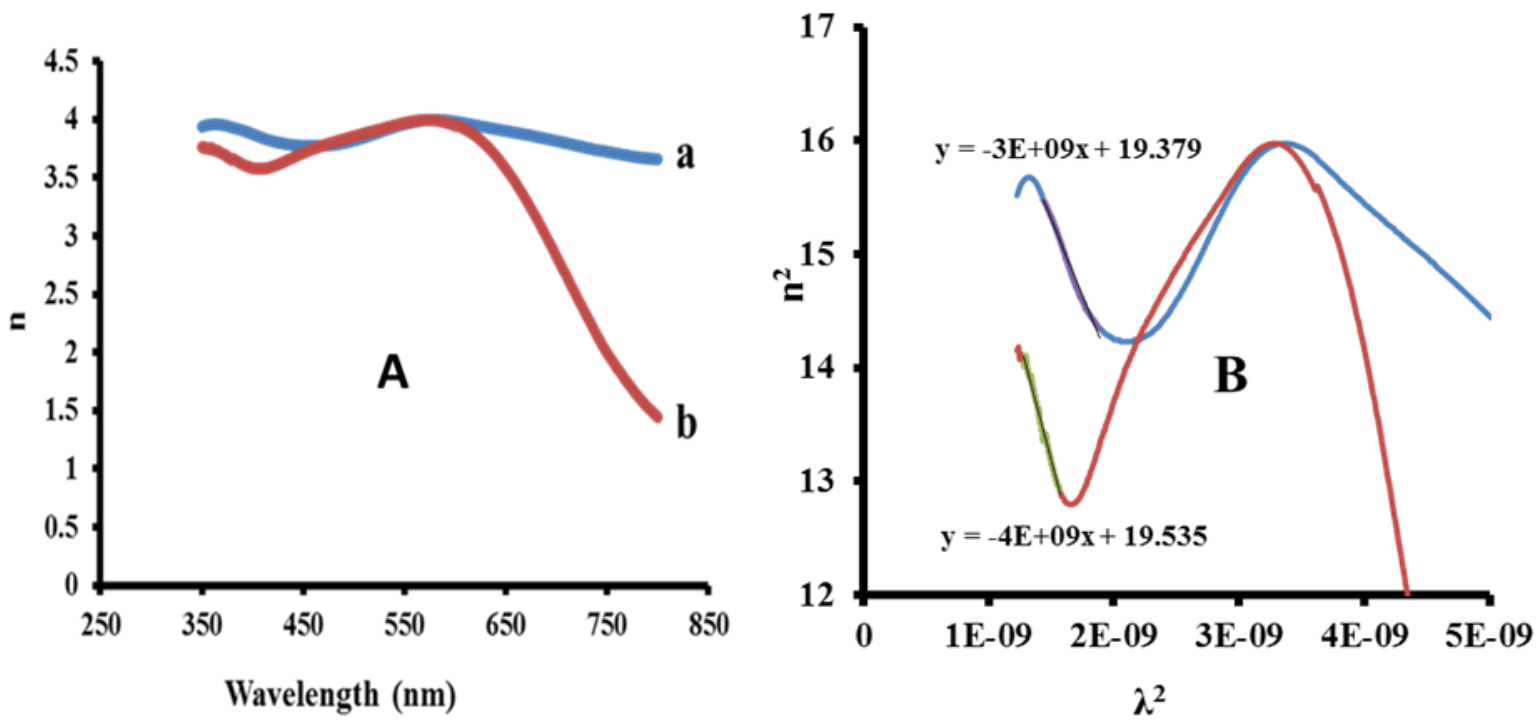

Figure 5. A) Refractive index $n$ vs wavelength, B) $n^{2}$ vs $\lambda^{2}$ for a) PBTh and b) PDBrBTh

Figure 5B was created using the following equation [Humar G.A. et al 2001):

$$
n^{2}=\varepsilon_{L}-\left[\frac{e^{2}}{\Pi C^{2}}\right]\left[\frac{N}{m^{*}}\right] \lambda^{2}
$$

The intercepts of the linear equations displayed in Figure 5B denote to $\varepsilon_{L}$ lattice dielectric constant. These intercepts are 19.379 and $19.535 \mathrm{eV}$ for $\mathrm{PBTh}$ and for PDBrBTh, respectively. We do not have any reference value to judge the accuracy of these calculated quantities or if this relation can be applied to amorphous molecular materials. However, these close values indicate that no changes took place in the lattice dielectric constant.

b) Dielectric constants (real $\varepsilon_{r}$, and imaginary $\varepsilon_{i}$ )

Figure 6 displays the plots of the calculated $\varepsilon_{\mathrm{r}}$ and $\varepsilon_{\mathrm{i}}$ against photon energy. The plot of the $\varepsilon_{\mathrm{r}}$ vs photon energy is displayed in Figure 6A. Figure 6B shows the change in $\varepsilon_{\mathrm{i}}$ vs photon energy The results displayed in Figure 6A show that the $\varepsilon_{\mathrm{r}}{ }^{\text {'s }}$ of PBTh and PDBrBTh thin films are closer around a photon energy range of 1.9 to $2.7 \mathrm{eV}$. Above and below this range the real dielectric part for PDBrBTh was less than that of PBTh.

The results displayed in Figure 6B show that the $\varepsilon_{\mathrm{i}}$ of PDBrBTh film is less than that calculated for the polymer PBTh within a photon energy range up to $1.9 \mathrm{eV}$. However, the $\varepsilon_{\mathrm{i}}$ of PDBrBTh exceeds that of PBTh. Such behavior can be explained considering that at low photon energy, the structure of $\mathrm{PDBrBTh}$ inhibits the energy dissipation process [Martin Dressel 2002), while at photon energy greater than $2.7 \mathrm{eV}$ the structure of the PDBrBTh enhanced dissipation energy. This is most likely because at this greater photon energy, the dielectric material underwent energy absorption due to the dipole motion influence.
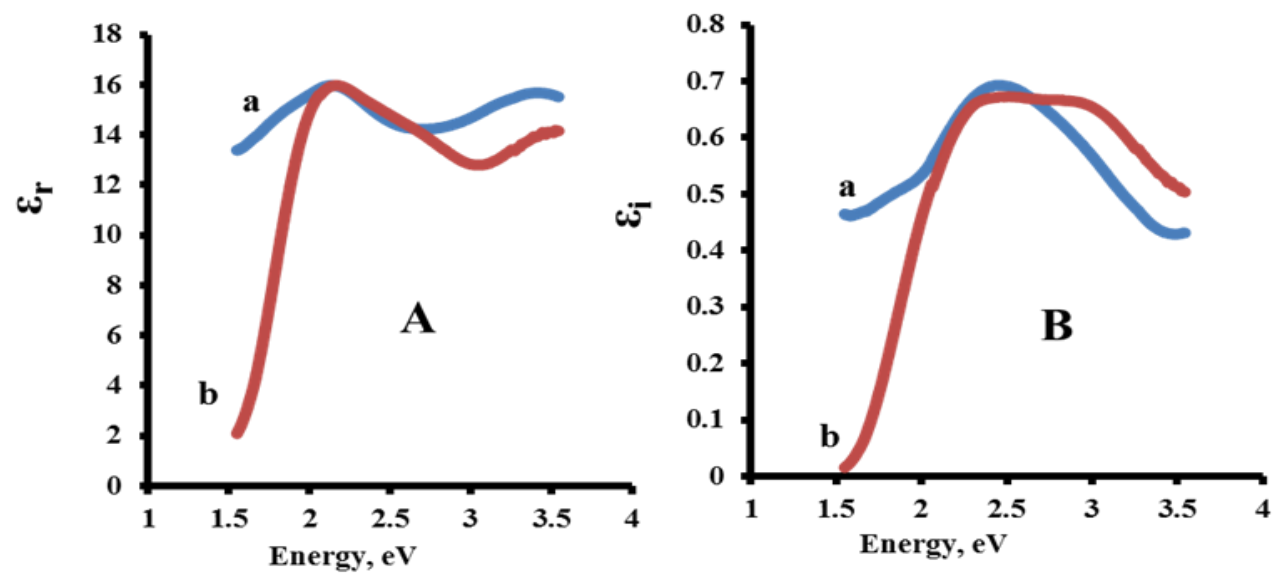

Figure 6. A) Real $\varepsilon_{\mathrm{r}}$, and B) imaginary $\varepsilon_{\mathrm{i}}$ components of dielectric constant for a) PBTh and b) PDBrBTh 
c) Optical conductivity $\left(\sigma_{\text {opt }}\right)$ and Electrical conductivity $\left(\sigma_{\text {ele }}\right)$

Both $\boldsymbol{\sigma}_{\text {opt }}$ and $\boldsymbol{\sigma}_{\text {ele. }}$ were calculated using the following formulas [Sharma P. Et al , 2007, Sabari G. et al 2009, Saranraj A et al 2017):

$$
\sigma_{\text {opt }}=\frac{\alpha n c}{4 \Pi}
$$

and

$$
\sigma_{e l e}=\frac{2 \lambda \sigma_{o p t}}{{ }_{\text {ol }}}
$$

The plots of $\boldsymbol{\sigma}_{\text {opt }}$ and $\boldsymbol{\sigma}_{\text {ele. }}$ vs photon energy for PBTh and for P\&B BrBTh are displayed in Figure 7.

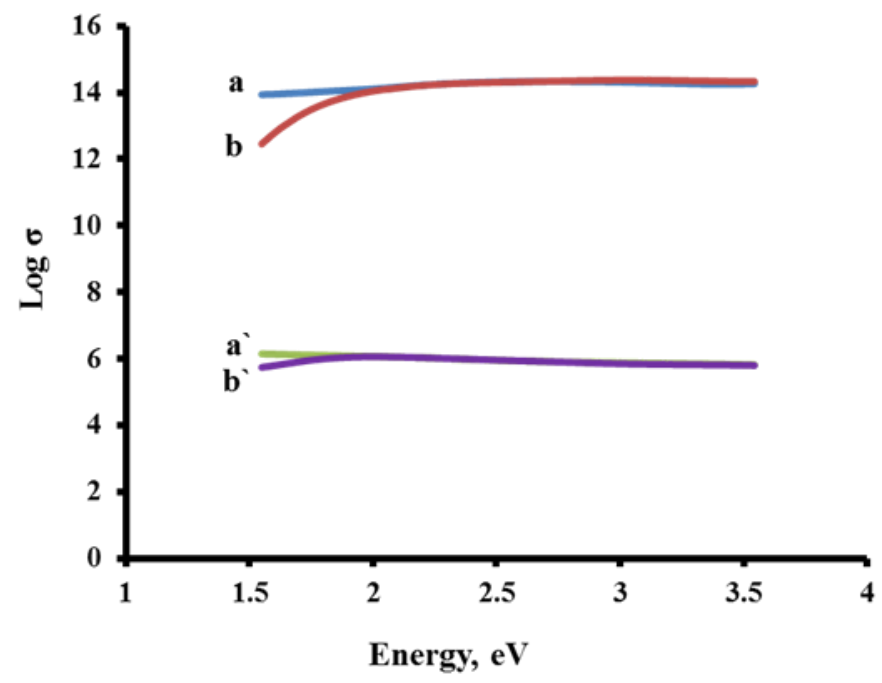

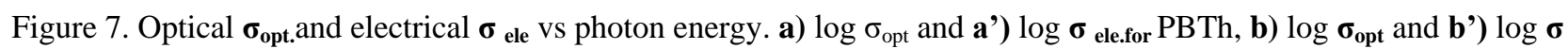
ele. for PDBrBTh

Figures $7 \mathrm{a}$ and $\mathrm{b}$ clearly show that 1) $\sigma_{\text {opt }}$ for PBTh is greater than that of PDBrBTh at photon energy up to $\approx 1.8 \mathrm{eV}$, and 2) $\sigma_{\mathrm{opt}}$ for both polymers has very close value. It known that optical conductivity is related to the light-electrical component and it is time dependent. The electrical component of the light varies its amplitude and direction over time. The lower optical conductivity of PDBrBTh compared to PBTh at a low photon energy can be explained on the basis that the presence of $\mathrm{Br}$ hinders the interaction of the polymer with the electric component of light. At a photon energy smaller than $1.8 \mathrm{eV}$, PDBrBTh lacks the ability to provide the additional charge transfer needed for the conductivity (Sharma P. et al, 2007). Figures 7 a' and b' indicates similar behavior to that observed in Figure 7 a',b' which also shows that $\sigma_{\text {ele. }}$ for PBTh, and for PDBrBTh are smaller than the corresponding $\sigma_{\text {opt. }}$. Such behavior can be explained on the basis of the Drude model (Drude Paul, 1900), as electrical conductivity is considered as optical conductivity in a static filed.

\subsection{Photoelectrochemical Behavior}

Unless otherwise noted, the photoelectrochemical behavior was investigated in the dark and under illumination by cycling the potential of FTO/ PBTh and FTO/ PDBrBTh between -1.0 to $1.0 \mathrm{~V} \mathrm{vs}$. $\mathrm{Ag} / \mathrm{AgCl}$ at a scan rate of $0.10 \mathrm{~V} / \mathrm{s}$ in a given electrolyte. The electrode surface area was kept at $2.0 \mathrm{~cm}^{2}$.

\subsubsection{Electrochemical Behavior in Aqueous Acetate Electrolyte}

Figure 8A displays the $\mathrm{CV}$ of both the FTO/PBTh and FTO/PDBrBTh assemblies in $0.2 \mathrm{M}$ acetate electrolyte (pH 8). This figure indicates that the recorded photocurrent for PBTh (Figure 8A-a) is greater than that of PDBrBTh (Figure $8 \mathrm{~A}-\mathrm{b})$. Greater photocurrent is a reflection of better charge separation. It is clear that PBTh facilitated charge separation and charge transfer more than PDBrBTh did. Figure 8B shows the photocurrent-time curve recorded in cycles of illumination and darkness in oxygenated electrolyte at $-0.5 \mathrm{~V}$ vs $\mathrm{Ag} / \mathrm{AgCl}$. It is noticed that PBTh again generated more photocurrent than PDBrBTh even in longer illumination periods. The fact that PBTh maintained constant high photocurrent current suggests that PBTh possesses greater photo stability. This means that PBTh (Figure 8B-a') is more photoactive than PDBrBTh (Figure 8B-b'). Photocurrent is generally produced when efficient charge separation occurs. These results suggest that very low electron/hole recombination $(\mathrm{e} / \mathrm{h})$ occurred in the illuminated PBTh. The existence 
of $\mathrm{Br}$ on the thiophene ring may contribute to the low charge separation and consequently to the low photocurrent.
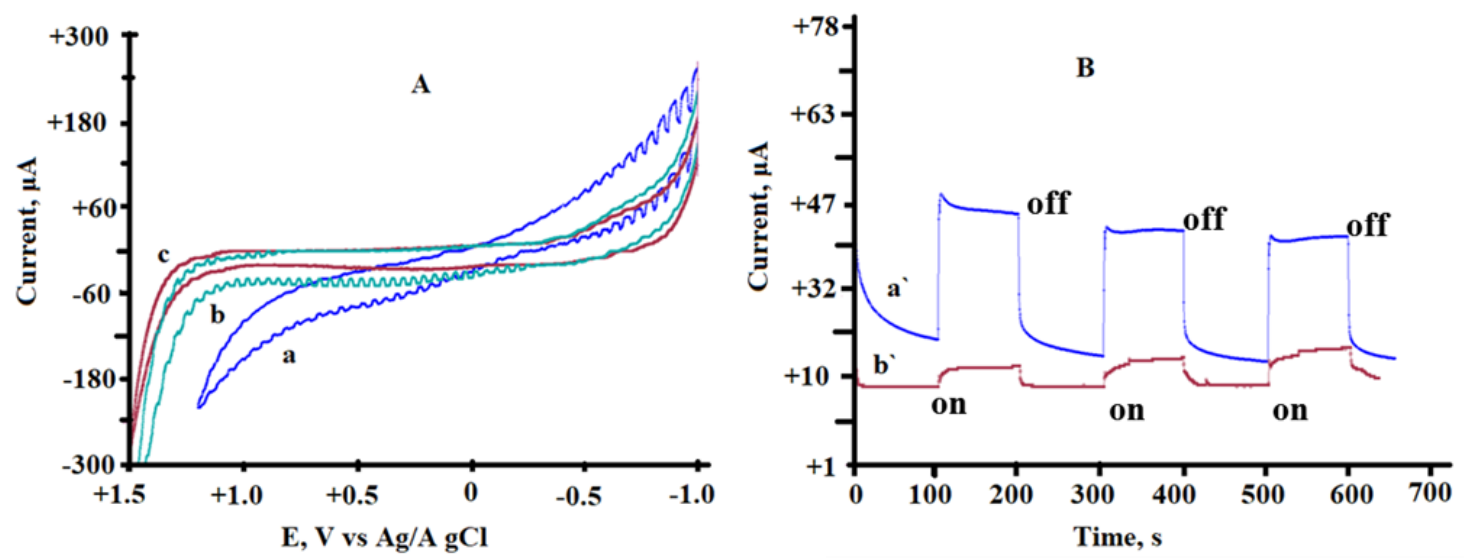

Figure 8. Photoelectrochemical behavior of in $0.2 \mathrm{M}$ acetate electrolyte under illumination A) $\mathrm{CV}$ at $0.1 \mathrm{~V} / \mathrm{s}$ in a) $\mathrm{PBTh}$, b) PDBrBTh, c) PDBrBTh in dark. B) Photocurrent vs time curve at $-0.5 \mathrm{~V}$ vs $\mathrm{Ag} / \mathrm{AgCl}$ in presence of $\mathrm{O}_{2}: \mathrm{a}^{\prime}$ ) $\mathrm{PBTh}, \mathrm{b}$ ') PDBrBTh

\subsubsection{Electrochemical Behavior in Aqueous Citrate Electrolytes}

Figure 9 displays the electrochemical behavior of FTO/PBTh and FTO/PDBrBTh assemblies in aqueous citrate electrolyte $(\mathrm{pH}$ 8). The behavior was similar to that observed in acetate electrolyte, except that PDBrBTh gave inconsistent photocurrent-time track (Figure 9 B-b'). PBTh gave a reproducible small sharp anodic current spike in the first few seconds of illumination. Such behavior is an indication of fast charge recombination due to hole accumulations at the outermost layers of the assembly/electrolyte interface (Sookhakian M. Et al 2014).
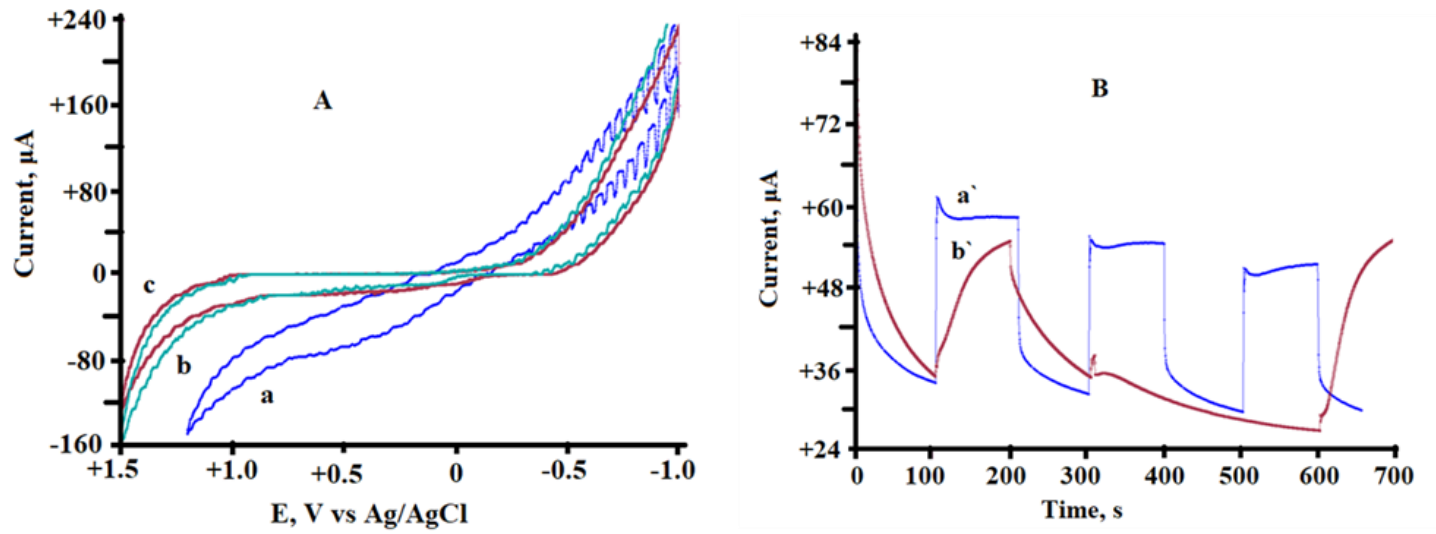

Figure 9. Photoelectrochemical behavior in $0.2 \mathrm{M}$ Citrate electrolyte ( $\mathrm{pH}$ 8) under illumination $\mathbf{A}) \mathrm{CV}$ at $0.1 \mathrm{~V} / \mathrm{s}$ a)PBTh, b)PDBrBTh, c) in dark B) Photocurrent vs time curve at $-0.5 \mathrm{~V}$ vs $\mathrm{Ag} / \mathrm{AgCl}$ in the presence of $\left.\mathrm{O}_{2}: \mathrm{a}^{\prime}\right) \mathrm{PBTh}$, and b') PDBrBTh.

\subsubsection{Electrochemical Behavior in Aqueous Tartrate Electrolytes}

The CV behavior of FTO/PBTh and FTO/PDBrBTh assemblies in aqueous tartrate electrolyte ( $\mathrm{pH} 8$ ) under illumination is displayed in Figure $10 \mathrm{~A}$. Such behavior is similar to that observed in acetate and citrate. However, Figure 10B-b' shows that PDBrBTh gives an inconsistent photocurrent-time track. Furthermore it shows that PBTh gives a large and reproducible sharp anodic current spike in the first few seconds of illumination. Comparing the magnitude of this photocurrent spike in tartrates to that observed in citrate indicates that PBTh/tartrate created greater hole accumulation than that in PBTh/citrate electrolyte. 

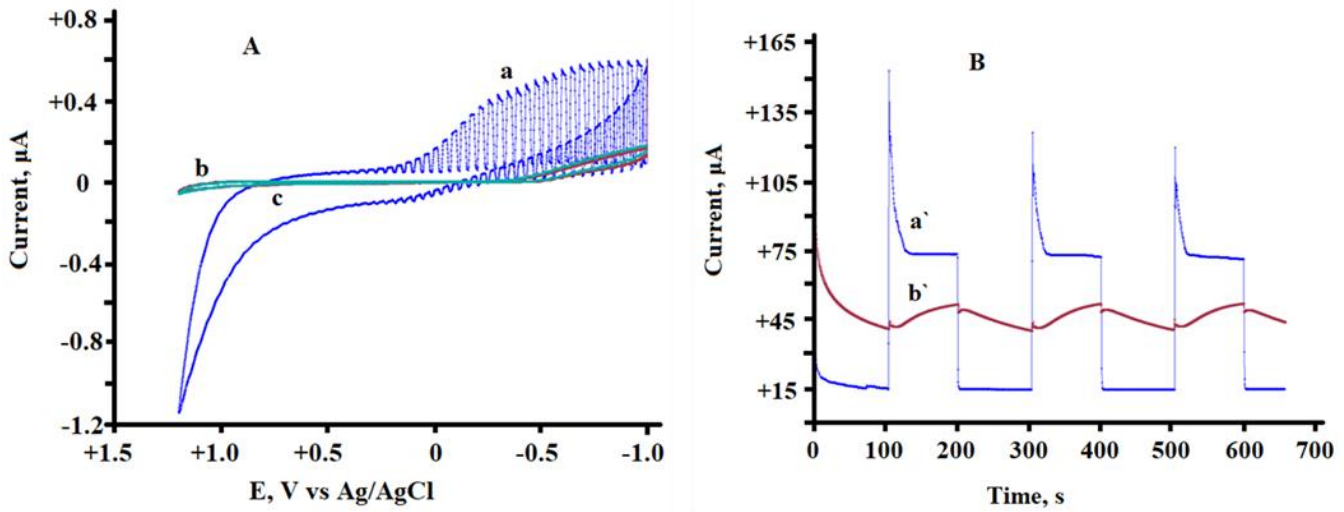

Figure 10. Photoelectrochemical behavior in $0.2 \mathrm{M}$ tartrate electrolyte ( $\mathrm{pH} 8)$ under illumination $\mathrm{A}) \mathrm{CV}$ at $0.1 \mathrm{~V} / \mathrm{s}$ a)PBTh, b)PDBrBTh c) in dark B) Photocurrent vs time curve at $-0.5 \mathrm{~V} \mathrm{vs} \mathrm{Ag} / \mathrm{AgCl}$ in the presence of $\left.\mathrm{O}_{2}: \mathrm{a}^{\prime}\right) \mathrm{PBTh}$, and b') PDBrBTh

\subsubsection{Electrochemical Behavior in Aqueous Phosphate Electrolytes}

Figure 11A displays the $\mathrm{CV}$ of both FTO/PBTh and FTO/PDBrBTh assemblies in aqueous phosphate electrolyte (pH 8) in the dark and under illumination. This figure shows that PDBrBTh gives much less photocurrent than PBTh, even under a long illumination time (Figure 11B). Furthermore, Figure 11B, shows that while PBTh gives a large and reproducible photocurrent that reflects hole accumulation (Figure 11B-a'). PDBrBTh gives an inconsistent and very low photocurrent-time track (Figure 11B-b').
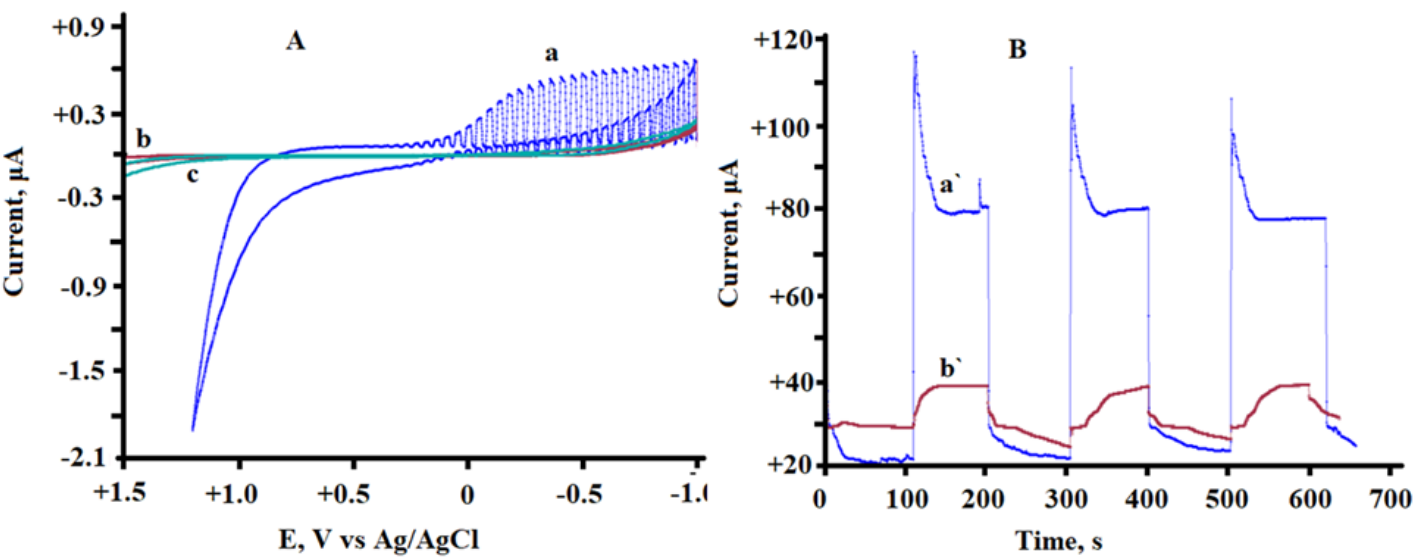

Figure 11. Photoelectrochemical behavior in $0.2 \mathrm{M}$ phosphate electrolyte (pH 8) under illumination. A) $\mathrm{CV}$ at $0.1 \mathrm{~V} / \mathrm{s}$ a)PBTh, b)PDBrBTh c) in dark B) Photocurrent vs time curve at $-0.5 \mathrm{~V}$ vs $\mathrm{Ag} / \mathrm{AgCl}$ in the presence of $\mathrm{O}_{2}: \mathrm{a}^{\prime}$ ) PBTh and b') PDBrBTh

\subsubsection{Electrochemical Impedance Spectroscopic Studies}

Impedance investigations for FTO/ PBTh and FTO/ PDBrBTh were performed and analyzed in a three-electrode cell containing liquid electrolytes, between $10^{5}-10^{-2} \mathrm{~Hz}$ utilizing Solartron 1201A, MX-studio ECS software. Sample of the impedance complexes (Nyquist plot) generated from these studies are displayed in Figure 12. This figure shows that PBTh, in neutral or doped form, possesses greater charge transfer resistance $\left(\mathrm{R}_{\mathrm{f}}\right)$ to the faradic process than does PDBrBTh. The doped and neutral PDBrBTh have the same $R_{f}$ values. However, only neutral PDBrBTh shows diffusional control region (Warburg impedance). The existence of the semicircle indicates that the charge transfer is kinetically controlled. EIS data analysis results in calculations of several physical properties of PBTh and PDBrPTh. Tables 1 and 2 are list of some impedance data for both PBTh and PDBrBTh. While Table 1 shows that relaxation times $\left(\tau=1 / \omega_{\max }\right)$ for PBTh are longer than those of PDBrBTh, Table 2 shows that PBTh possesses greater $\mathrm{C}_{\mathrm{dl}}$ (double layer capacitance) in both neutral and doped states than PDBrBTh. Only the PDBrBTh neutral film shows a Warburg diffusion region (Figure $12 \mathrm{Bb}$ ). No evidence of charge saturation was observed with the cell assemblies used in these studies. 

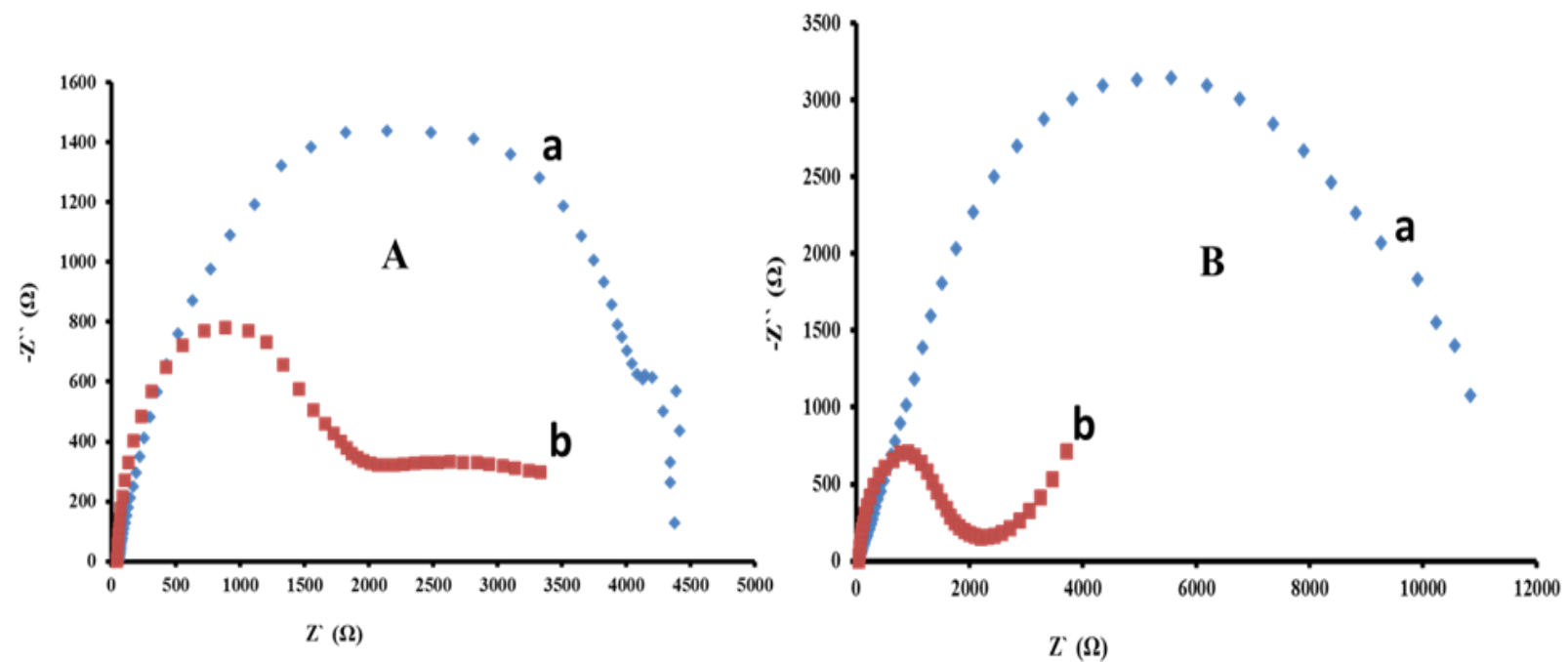

Figure 12. Nyquist plot of $1 \mu \mathrm{m}$ polymer films on FTO in $0.2 \mathrm{M}$ Citrate electrolytes (pH 6) under illumination. A) doped film at $0.9 \mathrm{~V}$ vs $\mathrm{Ag} / \mathrm{AgCl}$ and $\mathrm{B}$ ) neutral films at $-0.7 \mathrm{~V}$ vs $\mathrm{Ag} / \mathrm{AgCl}$ for a) $\mathrm{PBTh}, \mathbf{b}) \mathrm{PDBrBTh}$

Table 1. EIS data (relaxation time or PBTh and PDBrBTh

\begin{tabular}{|c|c|c|c|c|c|c|c|c|}
\hline \multirow[t]{3}{*}{ Electrolyte } & \multicolumn{4}{|c|}{ Relaxation time $(\tau)$ 's (PBTh) } & \multicolumn{4}{|c|}{ Relaxation time $(\tau)$ 's (PDBrBTh) } \\
\hline & \multicolumn{2}{|r|}{ Dark } & \multicolumn{2}{|r|}{ Light } & \multicolumn{2}{|r|}{ Dark } & \multicolumn{2}{|c|}{ Light } \\
\hline & Neutral & Doped & Neutral & Doped & Neutral & Doped & Neutral & Doped \\
\hline Acetate & 8 & 0.8 & 8 & 1.26 & 0.251 & 0.2 & 0.2 & 0.004 \\
\hline Citrate & 5.012 & 2.512 & 6.310 & 0.4 & 0.5 & 0.4 & 0.316 & 0.126 \\
\hline Phosphate & 5 & 0.05 & 8 & 0.08 & 0.316 & 0.316 & 0.5 & 0.16 \\
\hline Tartrate & 0.04 & 0.06 & 0.025 & 0.016 & 6.31 & & 0.316 & 0.5 \\
\hline
\end{tabular}

Table 2. EIS data (double layer capacitance) for PBTh and PDBrBTh

\begin{tabular}{|c|c|c|c|c|c|c|c|c|}
\hline \multirow[t]{3}{*}{ Electrolyte } & \multicolumn{4}{|c|}{$\mathrm{C}_{\mathrm{dl}}(\mathrm{PBTh}), \mu \mathrm{F}$} & \multicolumn{4}{|c|}{$\mathrm{C}_{\mathrm{dl}}(\mathrm{PDBrBTh}), \mu \mathrm{F}$} \\
\hline & \multicolumn{2}{|r|}{ Dark } & \multicolumn{2}{|r|}{ Light } & \multicolumn{2}{|r|}{ Dark } & \multicolumn{2}{|c|}{ Light } \\
\hline & Neutral & Doped & Neutral & Doped & Neutral & Doped & Neutral & Doped \\
\hline Acetate & 264 & & 397 & & 126 & & 133 & \\
\hline Citrate & 278 & 15 & 37 & 556 & 667 & 313 & 176 & 3968 \\
\hline Phosphate & 5 & 0.05 & & 0.08 & & & & \\
\hline Tartrate & 926 & 0.06 & 1600 & 0.02 & 4 & & 127 & \\
\hline
\end{tabular}

\subsection{Band-Energy Map of PBTh and PDBrBTh}

The measured onset oxidation potentials $E_{o x}$ for each PBTh and PDBrPTh (figure 2, 8-11) along with data obtained from Figure 3 were used to calculate the electron affinity (EA), band gap, and ionization potential (IP) following the formula previously published (Slawomir B. et al 2007). The positions of HOMO and LUMO of each polymer were approximatly identified. Figure 13 display the relative positions of energy bands for each polymer. This figure shows that 1) PDBrPTh has a larger band gap, greater HOMO level than PBTh, and 2) PDBrPTh possesses a smaller EA than $\mathrm{PBTh}$. We suggest that these two facts, alonge with the donor/acceptor character of $\mathrm{Br}$ atom on $\mathrm{C} 3$, contributed to the lower photoactivety of PDBrPTh than that of PBTh. 


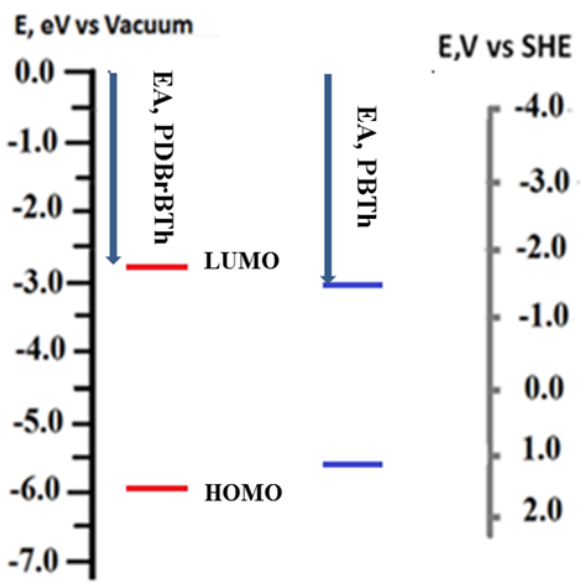

\section{Conclusion}

Figure 13. Energy map for PBTh and PDBrBTh

Studies show that introduction of bromine as a substituent at C-3 position of the thiophene rings, causes greater ionization potential (IP) and greater HOMO than PBTh. It also shows that both polymers have closer values around a photon energy range of 1.9 to $2.7 \mathrm{eV}$. At above and below this range, the real dielectric part for PDBrBTh was less than that of PBTh. Furthermore, lower optical conductivity and photoactvity were observed in PDBrBTh than in $\mathrm{Br}$-free PBTh at lower photon energy.

These observed properties suggest that bromine can be used to tune and alter the photoactivity and morphology of photoactive organic semiconductor. The polymer chain planarity can also be affected by the presence of $\mathrm{Br}$ atoms, compared with unsubstituted PBTH film.

\section{Acknowledgements}

The authors acknowledge School of Sciences at Indiana University Kokomo for its valuable support.

\section{References}

Braun, S., Osikowicz, W., Wang, Y., \& Salaneck, W. R. (2007). Energy level alignment regimes at hybrid organicorganic and inorganic-organic interfaces. Organic Electronics 8, 14-20. https://doi.org/10.1016/j.orgel.2006.10.006

Carbas, B. B. (2017). Novel electrochromic copolymers based on 3-3'-Dibromo-2-2 -Biothiophene and 3,4 Ethylene Dioxythiophene. J. Polymer, 113, 180-186. https://doi.org /10.1016/j.polymer.2017.02.053

Decher, G. (1997). Fuzzy Nanoassemblies: Toward Layered Polymeric Multicomposites. Science, 277, 1232. https://doi.org/10.1126/science.277.5330.1232

Drude, P. (1900). Zur Elektronentheorie der Metalle. Annalen der Physik. 306(3), 566. Bibcode: 1900AnP...306..566D. https://doi.org/10.1002/andp.19003060312

Druy, M. A., \& Seymour, R. J. (1983). Poly (2,2' - BITHIOPHENE): An Electrochromic Conducting Polymer. J. Phys. Colloques, 44, C3-595-C3-598. https://doi.org/10.1051/jphyscol:19833119

Kasem, K. K., Worley, H., \& Elmasry, M. (2018). Optical and Photoelectrochemical Studies on Photoactive Inorganic/Organic/Organic/ Interface Assemblies of CdS/Poly 3-(2-thienyl) aniline/ Poly2,2 Bithiophene. Advanced Composites and Hybrid Mat. https://doi.org/10.1007/s42114-018-0055-0

Kivrak, A., Yildiz, H. B., G“Okyer, S., \& Carbas, B. B. (2018). Electrochemical polymerization of new alkoxy-bridged dithieno (3,2-B:2',3'-D) pyrrole derivative. Turk. J. Chem., 42, 439-447. https://doi.org/10.3906/kim-1709-33

Kohsuke, K., \& Hiromasa, G. (2012). Synthesis and optical properties of 1,1-binaphthyl thiophene alternating copolymers with main chain chirality. Journal of Materials Chemistry, 22(44), 23514-23524. https://doi.org/10.1039/c2jm35594a

Kumar, G. A., Thomas, J., George, N., Kumar, B. A., Shnan, P. R., Npoori, V. P., \& Unnikishnan, N. V. (2001). Optical absorption studies of free $(\mathrm{H} 2 \mathrm{Pc})$ and rare earth $(\mathrm{RePc})$ phthalocyanine doped borate glasses. J. Phys Chem Glasses, 41, 89-93. 
Kuo, C. W., Wu, T. Y., \& Fan, S. C. (2018). Applications of Poly(indole-6-carboxylic acid-co-2,20-bithiophene) Films in High Contrast Electrochromic Devices. J. Coatings, 8,102. https://doi.org/10.3390/coatings8030102

Martin, D. (2002). Electrodynamics of Solids Optical Properties of Electrons in Matter”, Editor George Grüner, Cambridge university press, Cambridge, UK.

Ryu, D. Y., Shin, K., Drockenmuller, E., Hawker, C. J., \& Russell, T. P. (2005). A generalized approach to the modification of solid surfaces. Science, 308, 236. https://doi.org/10.1126/science.1106604

Sabari, G., \& Dhanushkodi, T. C. (2009). Linear and nonlinear optical properties of tris thiourea zincsulphate single crystals. Cryst. Res. Tech., 44(12), 1297-1302. https://doi.org/10.1002/crat.200900351

Saranraj, A, Sahaya, S., Jude, D., Vinitha, G., \& Martin, B. D. S. A. (2017). Third harmonic generation and thermos-physical properties of benzophenone single crystal for phototonic applications. Materials Research Express, 4, 106-204. https://doi.org/10.1088/2053-1591/aa8b7b

Sharma, P., \& Katyal, S. C. (2007). Determination of optical parameters of alfa $\left(\mathrm{As}_{2} \mathrm{Se}_{3}\right){ }_{90} \mathrm{Ge}_{10}$ thin film. Journal of Physics D: Applied Physics, 40(7), 2115-2120. https://doi.org/10.1088/0022-3727/40/7/038

Sookhakian, M., Amin, Y. M., Baradaran, S., Tajabadi, M. T., MoradiGolsheikh, A., \& Basirun, W. J. (2014). A layer-by-layer assembled graphene/zinc sulfide/polypyrrole thin-film electrode via electrophoretic deposition for solar cells. Thin Solid Films, 552, 204-211. https://doi.org/10.1016/j.tsf.2013.12.019

Tauc, J. (1968). Optical properties and electronic structure of amorphous Ge and Si. Materials Research Bulletin, 3, 37-46. https://doi.org/10.1016/0025-5408(68)90023-8

Teare, D. O. H., Spanos, C. G., Ridley, P., Kinmond, E. J., Roucoules, V., Badyal, J. P. S., ... Willis, C. (2002). Pulsed Plasma Deposition of Super-Hydrophobic Nanospheres. Chem. Mater., 14, 4566. https://doi.org/10.1021/cm011600f

Ulman, A. (1996). Formation and Structure of Self-Assembled Monolayers. Chem. Rev., 96, 1533. https://doi.org/10.1021/cr9502357

Urbach, F. (1953). The Long-Wavelength Edge of Photographic Sensitivity and of the Electronic Absorption of Solids. Phys. Review, 92, 1324. https://doi.org/10.1103/PhysRev.92.1324

Wei, Y., Chan, C. C., Tian, J., Jang, G. W., \& Hsueh, K. F. (1991). Electrochemical Polymerization of Thiophenes in the Presence of Bithiophene or Terthiophene: Kinetics and Mechanism of the Polymerization. J. Chem., Mater, 3(5), 888-897. https://doi.org/10.1021/cm00017a026

Wemple, S. H., \& DiDomenico, M. D. (1971). Behavior of the Electronic Dielectric Constant in Covalent and Ionic Materials. Phys. Rev. B, 3, 1338-1351. https://doi.org/10.1103/PhysRevB.3.1338

Zhang, X., \& Shen, J. C. (1999). Self-Assembled Ultrathin Films: From Layered Nanoarchitectures to Functional Assemblies. Adv. Mater., 11, 1139.

https://doi.org/10.1002/(SICI)1521-4095(199909)11:13<1139::AID-ADMA1139>3.0.CO;2-7

\section{Copyrights}

Copyright for this article is retained by the author(s), with first publication rights granted to the journal.

This is an open-access article distributed under the terms and conditions of the Creative Commons Attribution license (http://creativecommons.org/licenses/by/4.0/). 